\title{
Structure, Mechanism, and Application of Vacuum Insulation Panels in Chinese Buildings
}

\author{
Changhai Peng ${ }^{1,2,3}$ and Jianqiang Yang ${ }^{1}$ \\ ${ }^{1}$ School of Architecture, Southeast University, Nanjing 210096, China \\ ${ }^{2}$ Key Laboratory of Urban and Architectural Heritage Conservation, Southeast University, Ministry of Education, \\ Nanjing 210096, China \\ ${ }^{3}$ College of Engineering and Applied Science, University of Colorado Denver, Denver, CO 80217, USA \\ Correspondence should be addressed to Changhai Peng; pengchanghai@foxmail.com
}

Received 2 January 2016; Revised 27 March 2016; Accepted 19 April 2016

Academic Editor: Ana S. Guimarães

Copyright (C) 2016 C. Peng and J. Yang. This is an open access article distributed under the Creative Commons Attribution License, which permits unrestricted use, distribution, and reproduction in any medium, provided the original work is properly cited.

Thermal insulation is one of the most used approaches to reduce energy consumption in buildings. Vacuum insulation panels (VIPs) are new thermal insulation materials that have been used in the domestic and overseas market in the last 20 years. Due to the vacuum thermal insulation technology of these new materials, their thermal conductivity can be as low as $0.004 \mathrm{~W} /(\mathrm{m} \cdot \mathrm{K})$ at the center of panels. In addition, VIPs that are composites with inorganic core and an envelope out of commonly three metallized PET layers and a PE sealing layer can provide B class fire resistance (their core materials are not flammable and are classified as A1). Compared with other conventional thermal insulation materials, the thermal insulation and fire resistance performances form the foundation of VIP's applications in the construction industry. The structure and thermal insulation mechanism of VIP and their application potential and problems in Chinese buildings are described in detail.

\section{Introduction}

Currently, building energy consumption in China has remained extremely high, reaching $33 \%$ of the total social energy consumption. In particular, the energy consumption through enclosure structures accounts for over $50 \%$ of the building energy consumption. To reduce building energy consumption, thermal insulation materials have received widespread applications. Due to their low thermal conductivity, organic thermal insulation materials have enjoyed particularly wide adoption. However, in recent years, frequent fire accidents have occurred due to these organic thermal insulation materials, for example, the Beijing Television Cultural Center fire [1] and the 2010 Shanghai fire [2]. Therefore, a thermal insulation material that combines High-Performance Thermal Insulation with fire resistance is urgently needed in the current Chinese building market.

VIPs (vacuum insulation panels) are inorganic composite thermal insulation panels with a thermal conductivity as low as $0.004 \mathrm{~W} /(\mathrm{m} \cdot \mathrm{K})$ at the center of panels [3]. The fire behavior of core materials is dependent on the types of fibers used for structural binding in the fumed silica core. Fumed silica core VIPs are class A, but the polymer barrier material is combustible [4]. However, additional layers added can reduce the behavior in the fire tests and one can imagine that it enables structural panels to reach one-hour fire rating. Thus, these materials can meet both requirements of highefficiency thermal insulation and fire resistance performance. Silica core VIPs primarily consist of thermal insulation with a porous rigid core and membrane wall, as shown in Figure 1. However, glass fiber VIPs are commonly added with getters. The rigid core provides support for VIP against atmospheric pressure; the membrane wall maintains the vacuum inside VIP, and the getters collect gases either leaked through the membrane or off gassed from the membrane materials [5].

In the 1980s, Brown, Boverie \& Cie (BBC) in Heidelberg, Germany, investigated rectangular evacuated casings filled with powders and fiber mats to insulate sodium-sulfur hightemperature batteries [6]. Applications for VIP emerged in refrigerators, freezers, shipping, and aerospace and other 


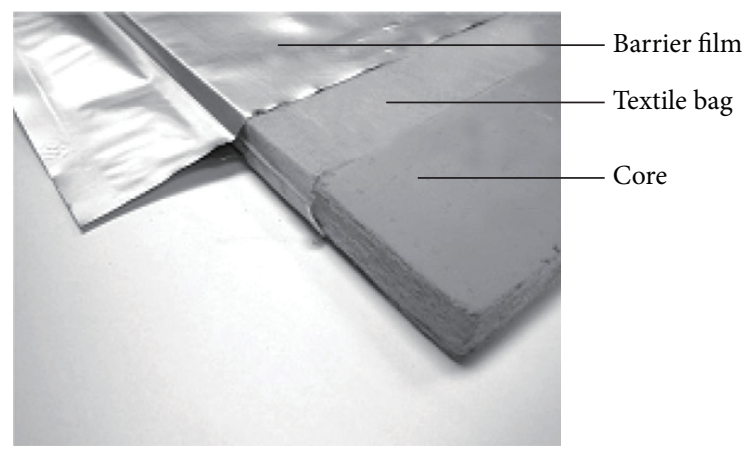

FIGURE 1: VIP [13].

industries. VIPs were first applied in the building sector in Germany and Switzerland in 2001 [7]. To date, the application of VIP in the building industry has lasted approximately 15 years. The preparation of materials, production of panels, characterization of performance, and application in buildings of VIP have been investigated by different countries around the world. For example, in Germany there have been a lot of test projects with constructions implementing VIPs, both refurbished and new constructions. Some were built as early as 2001 and have been monitored on a regular basis since then. Bayerisches Zentrum für Angewandte Energieforschung (ZAE Bayern) in collaboration with various VIP producers have many interesting projects which show how the implementation of VIPs into buildings has proceeded. ZAE Bayern conducted a research project called VIP Prove, where the aim was to see how VIPs behaved under practical conditions. To choose these projects, ZAE Bayern had certain criteria which the buildings had to fulfil, giving them a score of up to 85 points, where the higher the score was, the more suitable the construction was for monitoring $[6,8]$. Mandilaras et al. investigated the actual in situ hygrothermal performance of a full scale envelope insulated initially with conventional ETICS using expanded polystyrene (EPS) as an insulation material [9]. Johansson et al. explored how VIP can be used when retrofitting listed buildings to improve the thermal transmittance and moisture performance of the walls and the thermal comfort for the occupants [10]. Examples of a number of different constructions where VIP has been used in retrofitted building envelopes have been reported in the literature. During 20022005, the international efforts in VIP research were assembled in the IEA/ECBCS Annex 39 High-Performance Thermal Insulation (HiPTI). The project included monitoring and evaluation of 20 buildings with VIP in floors, roofs, walls, dormer windows, and other constructions [11]. In China, the government had also published the "Building Industry Standard of China" for "vacuum insulation panels for buildings" (JG/T 438-2014) on June 30, 2014 [12].

\section{Structure of VIP}

2.1. Core. The materials of the VIP core should possess certain characteristics. First, the materials should be porous, and the pore sizes should be small such that the contact points can be small; as a result, the thermal conduction will be reduced. Figure 2 shows the cylindrical glass fibers, and Figure 3 shows the spherical gas-phase silica. Second, the materials should not fracture under high external loading. Because a pressure of 1 mbar must be maintained inside the core, the prestress of the VIP should be approximately $100 \mathrm{kN} / \mathrm{m}^{2}$.

Currently, the primary types of VIP cores include foams, fibers, powders, and fiber-powder composites.

Foam polymers are a type of porous foam that are lightweight, thermal insulating, sound absorbing, shockproof, and corrosion resistant [14]. Polyurethane foam has received widespread application as VIP core material. It exhibits low thermal conductivity (thermal conductivity 20$30 \mathrm{~mW} / \mathrm{m} \cdot \mathrm{K}$ under nonvacuum) and is lightweight, easily produced, and inexpensive [15].

Fiber is a high-performance inorganic material that exhibits multiple qualities, for example, noncombustibility, nontoxicity, corrosion resistance, low density, low thermal conductivity, high insulation performance, and excellent chemical stability. Fiber is produced by two main processes: centrifugal spinning and jet firing. Panels made from fibers can be used as thermal insulation materials with thermal conductivity of $32-40 \mathrm{~mW} / \mathrm{m} \cdot \mathrm{K}$ [16]. As the material of a VIP core, the primary parameters of the fibers are type and diameter. VIP cores currently use mineral fibers and glass fibers, as shown in Figure 2. However, there are some safety and health concerns on glass fibers if they are less than 3 micrometers in diameter and greater than 20 micrometers in length [17].

The powders used in VIP cores are all inorganic, nonmetallic materials, including expanded perlite, lightweight pumice, and silica. Expanded perlite is an insulation material with low thermal conductivity. At atmospheric pressure and a temperature of 77-293 K, its mean thermal conductivity is $18.5-29 \mathrm{~mW} / \mathrm{m} \cdot \mathrm{K}$ [18]. As a VIP core material, expanded perlite exhibits advantages such as low cost. However, as a powder itself, it is extremely difficult to process and form shapes. In addition, the core is brittle and easily fractures even after forming shapes. As a VIP core material, silica includes fumed silica (also known as pyrogenic silica), precipitated silica, and silica aerogel. The first is prepared by the combustion method, whereas the latter two types are prepared by solution-phase synthesis. They all exhibit nanopore structures and therefore can reduce the thermal conductivity of gases. Figure 3 shows the fumed silica [13]. In Europe, fumed silica core VIPs have been professionalized and they have been better adapted to the needs of building applications because their aging and durability statements had been done [4].

To reduce the cost of VIP cores, a low-cost composite core material was investigated in 2009 by the National Research Council Institute for Research in Construction (NRC-IRC) [19]. This core material consisted of multilayer structures of pumice and glass fiber panels (Figure 4). Two products with densities of $340 \mathrm{~kg} / \mathrm{m}^{3}$ and $320 \mathrm{~kg} / \mathrm{m}^{3}$ for the core materials were manufactured.

Fiber-powder composites are a type of core material. Because this core material contains fiber layers, certain 


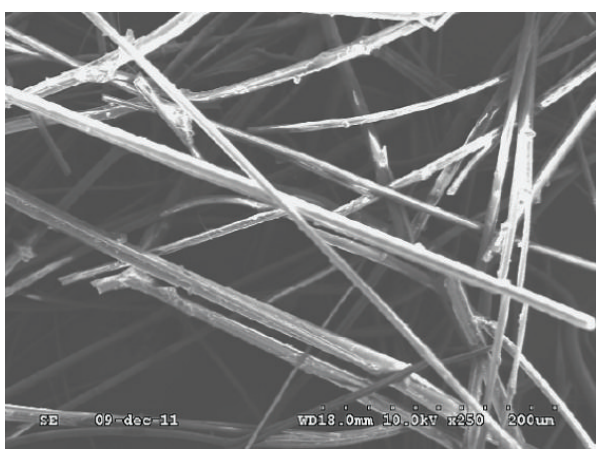

(a)

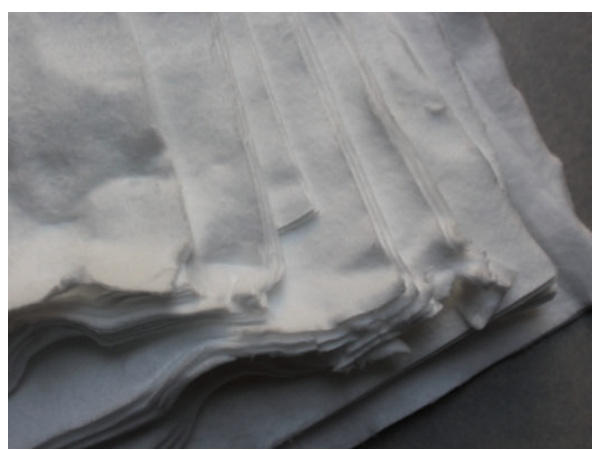

(b)

FIGURE 2: Scanning electron image (a) [13] and real image (b) (taken by authors) of glass fibers. Left image is a SEM picture of glass fiber insulation. The shape of the glass fibers is cylindrical which lead to small areas of contacts and thus small conductive heat transfer even if the material is compressed [13].

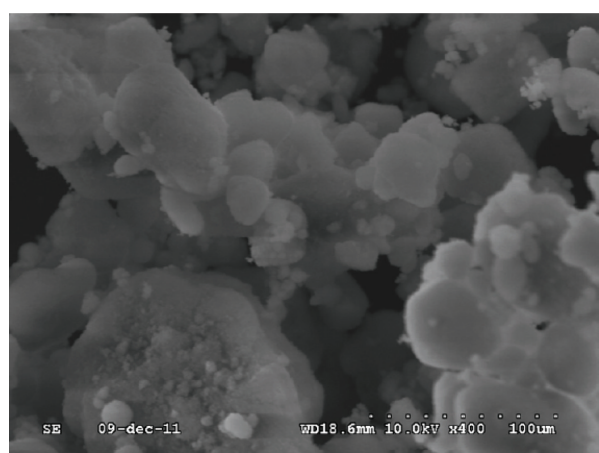

(a)

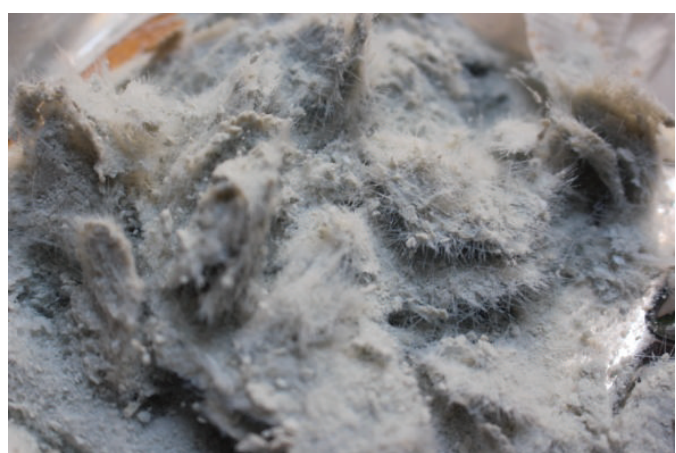

(b)

FIGURE 3: Scanning electron image (a) [13] and real image (b) of silica powders (taken by authors).

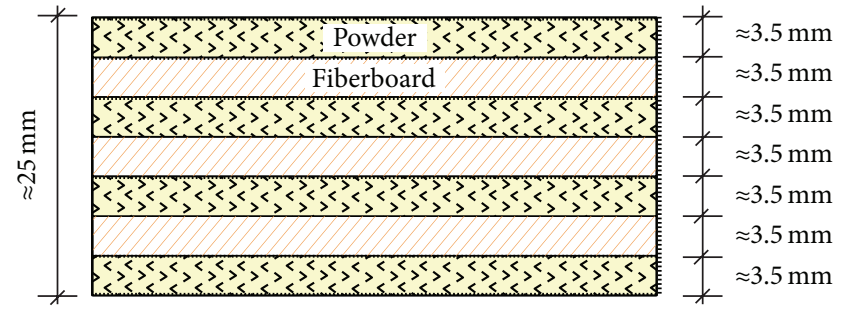

FIGURE 4: Structure of the composite core investigated by NRC-IRC [19].

undesired situations, such as the restoration of the core to its original shape, can result, most commonly due to gas leakage through the membrane. If VIP made from these core materials is applied to the walls of buildings, the leakage through the membranes can lead to the surface being peeled off from the walls. Therefore, the application of these products requires further investigations.

In summary, the stringent requirement for high vacuum, the negative environmental impact, and the flammability of the foam-type cores restrict their application in the thermal insulation of building walls. Although fibers possess low thermal conductivity, this VIP core material requires high vacuum. Additionally, when the vacuum disappears, the fibers will cause undesired effects, such as bulging walls. Although the conductivities of powders are higher than those of other types of core materials, powders receive more attention due to their related long expected durability. The advantages and disadvantages of different core materials are shown in Table 1.

2.2. Membrane. The primary function of membranes is to prevent air in the external environment from entering the core and thus to maintain a high vacuum inside. Once gases enter the inner core, the inner pressure increases, which raises the thermal conductivity of the inner core. When the thermal conductivity reaches certain values, if the transient working life is used, the material reaches the end of its working life. The thickness of the VIP membrane is normally 100-200 $\mu \mathrm{m}$. VIP membranes are often divided into the sealing layer, the barrier layer, and a protective layer as seen in Figure 5 [20]. These layers are described by Alam et al. [21] and Brunner et al. [22]. The inner layer is the sealing layer. This layer seals the core material in the envelope and traditionally consists of low- or high-density polyethylene (PE). The laminate surfaces are heat-sealed by two hot bars under pressure to bond 
TABLE 1: Characteristics of different core materials.

\begin{tabular}{lcll}
\hline Type & Representative material & Advantages & Disadvantages \\
\hline Fiber & Glass fiber & $\begin{array}{l}\text { Low thermal conductivity, easy } \\
\text { processing of ultrathin fibers, stable size, } \\
\text { allowable contraction and expansion, } \\
\text { being nonhygroscopic, and recoverability } \\
\text { after contact with water }\end{array}$ & $\begin{array}{l}\text { Caution during packaging, requirement } \\
\text { of high vacuum }\end{array}$ \\
\hline Foam & Foam polymer & $\begin{array}{l}\text { Low thermal conductivity, lightweight, } \\
\text { waterproofing }\end{array}$ & $\begin{array}{l}\text { Requirement of instruction from } \\
\text { technical staff, large contraction and } \\
\text { expansion, service temperature below } \\
350 \mathrm{~K}\left(77^{\circ} \mathrm{C}\right), \text { flammable }\end{array}$ \\
\hline \multirow{2}{*}{ Powder } & Gas-phase silica, expanded perlite & $\begin{array}{l}\text { Low thermal conductivity, low density, } \\
\text { low cost, fire retardant, nontoxicity, easy } \\
\text { to achieve vacuum }\end{array}$ & $\begin{array}{l}\text { Nonelasticity under pressure, difficulty to } \\
\text { mold }\end{array}$ \\
\hline
\end{tabular}
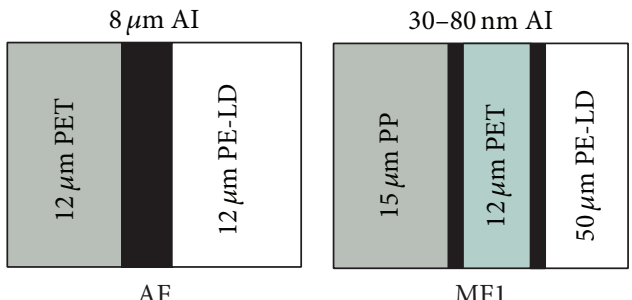

MF1

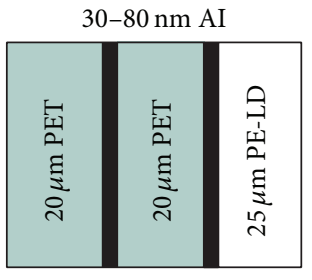

MF2

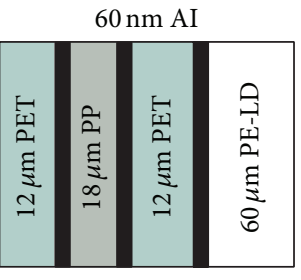

MF3

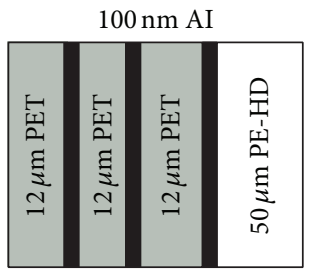

MF4

Figure 5: Illustration of an aluminum foil laminate (AF) and metallized polymer film MF [20].

together. The middle layer is the barrier layer in the case of the aluminum foil laminate (AF) type (Figure 5) [20]. Furthermore, metallized polymer film multilayer laminates (MF) are in common usage, where the metallized coating is typically deposited on a polyethylene terephthalate (PET) film (Figure 5) [20]. The purpose of the barrier layer is to prevent water vapor and air transmission through the envelope and into the VIP core. An outer protective layer may be added, for example, for improving the fire resistance properties, and may consist of glass fiber fabric or transparent lacquer. Environmental and handling stresses may damage the panel, so sometimes an additional protective layer aims to make the panel more robust, for example, applying expanded polystyrene (EPS), extruded polystyrene (XPS), or rubber granule layers or hard polymeric plates. The material chosen for the envelope should also be able to withstand general handling through transportation and installation without tearing. The common PET layer also works as a substrate for the barrier layer due to its superior flatness for the metallization process (coating) [20].

The membrane is the most important parameter in maintaining a long working life for the VIP. The evaluation of VIP membrane materials includes the penetration rates of gases, including those of oxygen and water vapors. The structure of the membrane material greatly affects the transmission of gases; different structures give rise to different transmission speeds. Multilayered foil coated membranes exhibit low thermal conductivity, but the penetration speed of gases is relatively high; by contrast, the penetration speed of gases is relatively low for foil layers, but the thermal conductivity is high. In summary, the application of VIP membranes requires the evaluation of synergistic effects of the foil layer and polymer layer.

The penetration rate of the air barrier should be small; thus, the pyrogenic silica core VIP can last 30 to 50 years and even up to 100 years in top quality building envelopes. The International Energy Agency (IEA) has noted in its 2005 report that the penetration rate of oxygen should be controlled within the range of $0-2 \mathrm{~cm}^{3} /\left(\mathrm{m}^{2}\right.$.day.bar) [7]. The valve depends on the size of the VIP and can only be used as an empirical value. If the inner core loses vacuum, the inner pressure will equalize with the outside atmospheric pressure, and the thermal conductivity will increase to $0.020 \mathrm{~W} /(\mathrm{m} \cdot \mathrm{K})$ for pyrogenic silica type of VIP cores.

2.3. Getter. A getter is a material that exhibits specific activity towards certain gases under certain conditions. To obtain a vacuum for the inner part of the VIP, the inner core is encapsulated by membrane materials. In a glass fiber core VIP with high vacuum requirement, getters are required to collect and remove gases because fiber core pore size is bigger than that of the fumed silica core type. The gases that penetrate to the core of the VIP primarily include $\mathrm{N}_{2}, \mathrm{O}, \mathrm{H}_{2}, \mathrm{CO}_{2}$, and $\mathrm{H}_{2} \mathrm{O}$. Water vapor can be removed by low-cost $\mathrm{CaSO}_{4}$ and $\mathrm{CaO}$; gases such as $\mathrm{O}_{2}, \mathrm{H}_{2}, \mathrm{CO}_{2}$, and $\mathrm{N}_{2}$ can be removed by active metals, such as barium, zirconium, and the alloys of these metals. Notably, these precious metals can complex or react with water, which decreases their capacity for gas absorption. Therefore, the getter apparatus is designed to remove water vapor first and then other gases. 


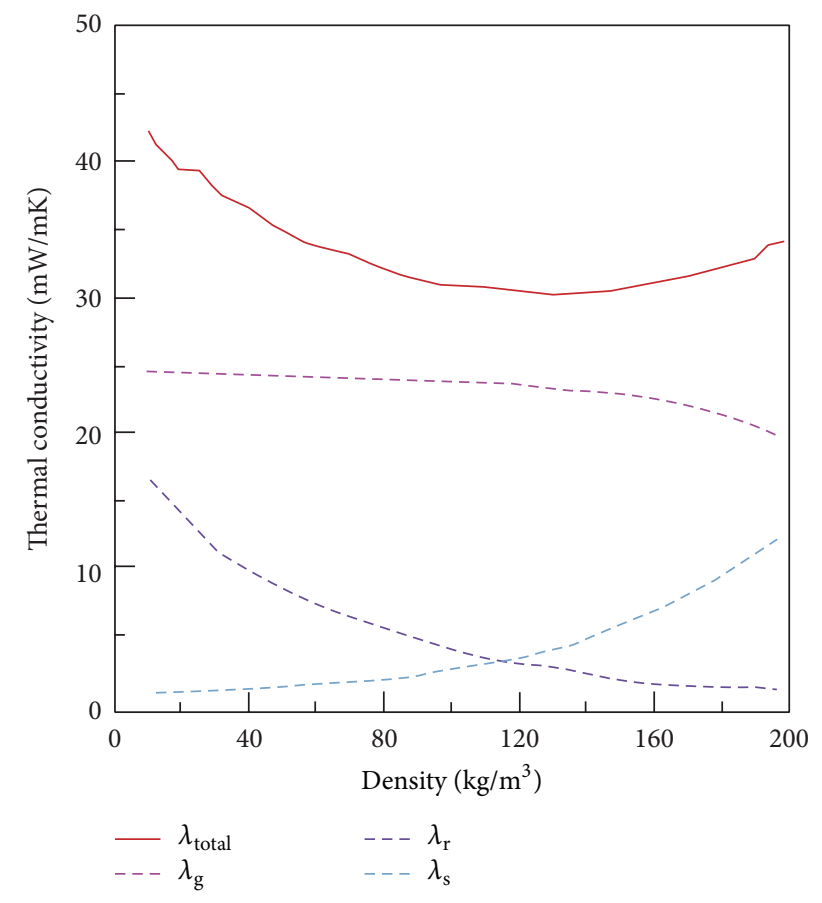

Figure 6: Comparison of the three heat transfer mechanisms in conventional thermal insulation materials [7].

\section{Thermal Insulation Mechanism of VIP}

In conventional thermal insulation materials, the contributions of the three heat transfer mechanisms to the thermal conductivity vary. As shown in Figure 6, solid heat transfer increases linearly with an increase in the bulk density. In contrast, radiation transfer decreases with an increase in the bulk density; for example, when the density is approximately $200 \mathrm{~kg} / \mathrm{m}^{3}$, the increase in the thermal conductivity due to radiation transfer is approximately $1-3 \mathrm{~mW} / \mathrm{m} \cdot \mathrm{K}$. Finally, gas heat transfer is responsible for the majority of the total heat transfer, with values between 20 and $30 \mathrm{~mW} / \mathrm{m} \cdot \mathrm{K}$. Therefore, if the gas heat transfer is decreased, the thermal conductivity of the materials will decrease dramatically. This relationship explains why special vacuum processing is used in VIP.

The total thermal conductivity of the VIP inner core can be described as

$$
\lambda_{\mathrm{C}}=\lambda_{\mathrm{S}}+\lambda_{\mathrm{R}}+\lambda_{\mathrm{G}}+\lambda_{\mathrm{C} v}+\lambda_{\text {coup }}
$$

where $\lambda_{\mathrm{S}}$ is the solid heat transfer $(\mathrm{W} /(\mathrm{m} \cdot \mathrm{K})), \lambda_{\mathrm{R}}$ is the radiation heat transfer $(\mathrm{W} /(\mathrm{m} \cdot \mathrm{K})), \lambda_{\mathrm{G}}$ is the gas heat transfer $(\mathrm{W} /(\mathrm{m} \cdot \mathrm{K})), \lambda_{\mathrm{C} v}$ is the gas convection inside the openings $(\mathrm{W} /(\mathrm{m} \cdot \mathrm{K}))$, and $\lambda_{\text {coup }}$ is the heat transfer from the coupled effect $(\mathrm{W} /(\mathrm{m} \cdot \mathrm{K}))$.

3.1. Solid Heat Transfer. Solid heat transfer in the core materials occurs on the necks through the physical contact between particles. The magnitude of this transfer is determined by the structure, density, and external pressure of the materials. The following equation expresses the relationship between

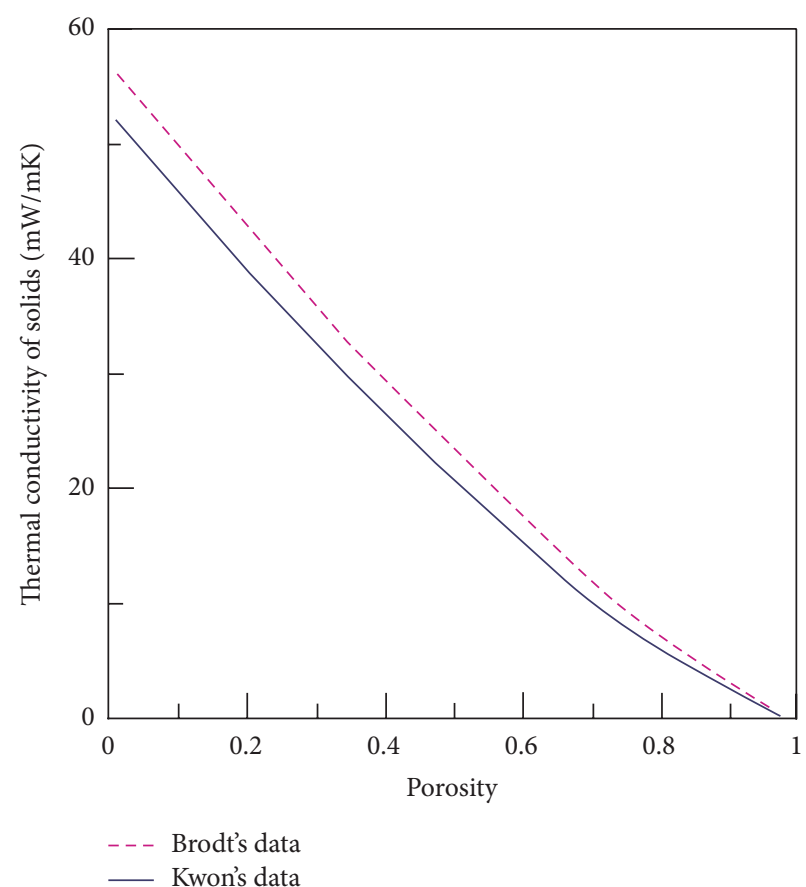

FIGURE 7: Relationship between the porosity and thermal conductivity of solids for VIP core materials.

the thermal conductivity of solids and the density of materials [23]:

$$
\lambda_{\mathrm{S}}=\rho^{\alpha}
$$

where $\rho$ is the density $\left(\mathrm{kg} / \mathrm{m}^{3}\right)$ and index $\alpha$ is a constant for foam materials and $1.5-2 \mathrm{~nm}$ class materials.

It can be seen from (2) that the smaller the density, the smaller the thermal conductivity of the solids.

Gas-phase silica will be used as an example of a VIP core material; the powder is assumed to consist of spherical particles. The decrease in $\lambda_{\mathrm{S}}$ can be explained in two ways. First, for the close-packed spherical particles, the orientation of contact between two spherical particles is different from the normal direction of the boundary spherical particles, which results in the tortuosity of heat transfer and the increase in the number of heat transfer pathways. Second, each contact between the spherical particles is a point contact, which increases the thermal resistance [14]. Brodt [24] and Kwon et al. [14] reported that the porosity of core materials also has a large effect on the thermal conductivity of solids, as shown in Figure 7. The figure shows that maintaining high porosity (i.e., low density) can further decrease the thermal conductivity of solids for the core materials.

3.2. Gas Heat Transfer. The gas heat transfer is referred to as the sum of gas conduction transfer and convection transfer. Its magnitude is determined by the mean free path of gas and the ratio of the path to the material pore size. Kaganer [25] 


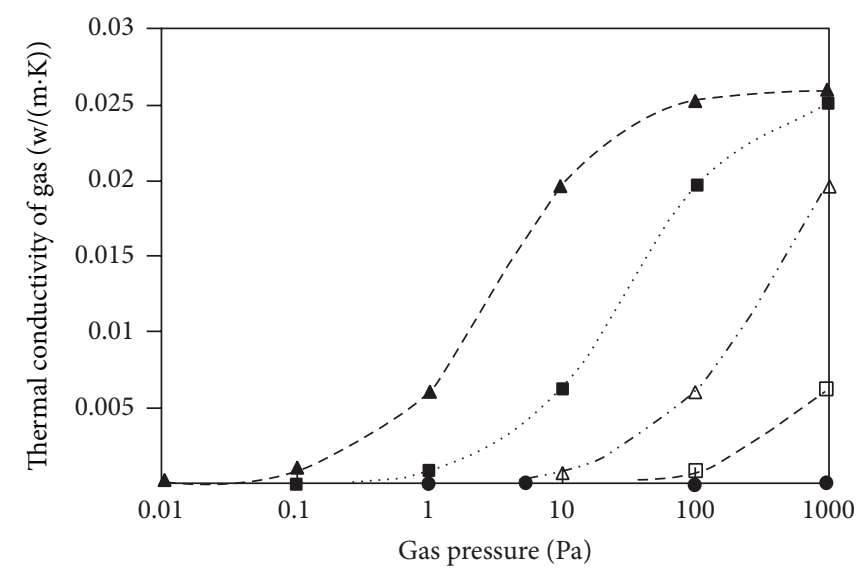

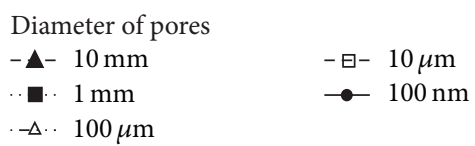

FIGURE 8: Relationship between the gas thermal conductivity and the atmospheric pressure and pore diameter [14].

proposed the following equation to calculate the gas thermal conductivity $\lambda_{\mathrm{G}}$ :

$$
\lambda_{\mathrm{G}}=\frac{\lambda_{0}}{(1+2 \beta \mathrm{Kn})},
$$

where $\lambda_{0}$ denotes the thermal conductivity of air under atmospheric pressure $[\mathrm{W} /(\mathrm{m} \cdot \mathrm{K})], \beta$ is an index that combines the activity coefficient and the inert coefficient of gases, and Kn denotes the Knudsen coefficient, where its value is the ratio of the mean free path of the gas to the diameter of the pores and can be represented by

$$
\mathrm{Kn}=\frac{1}{\Phi}=\frac{\left(k_{\mathrm{B}} T\right)}{\left(\sqrt{2} \phi d^{2} p \Phi\right)},
$$

where $k_{\mathrm{B}}$ is the Boltzmann constant $\left(1.38 \times 10^{-23} \mathrm{JK}^{-1}\right), T$ is the thermodynamic temperature $(\mathrm{K}), d$ is the diameter of molecules $(\mathrm{m})$, and $P$ is the gas pressure $(\mathrm{Pa})$.

Kwon et al. [14] proposed the following equation to calculate the gas thermal conductivity of air at $25^{\circ} \mathrm{C}(\beta=$ $0.0016 / P)$ :

$$
\lambda_{\mathrm{G}}=\frac{\lambda_{0}}{(1+(0.0032 / P \Phi))},
$$

where $P$ denotes the gas pressure $(\mathrm{Pa})$ and $\Phi$ is the pore size of the porous thermal insulation material $(\mathrm{m})$.

From (5), the relationship between the gas thermal conductivity of different porosity and pressure can be calculated, as shown in Figure 8. It can be seen from Figure 8 that, for the materials whose pore size is in the nanometer range, their thermal conductivity under atmospheric pressure can be neglected. However, it cannot be ignored under large pressure such as $10^{5} \mathrm{~Pa}$. Additionally, as the pore size increases, smaller pressure is required to maintain the small gas thermal conductivity.

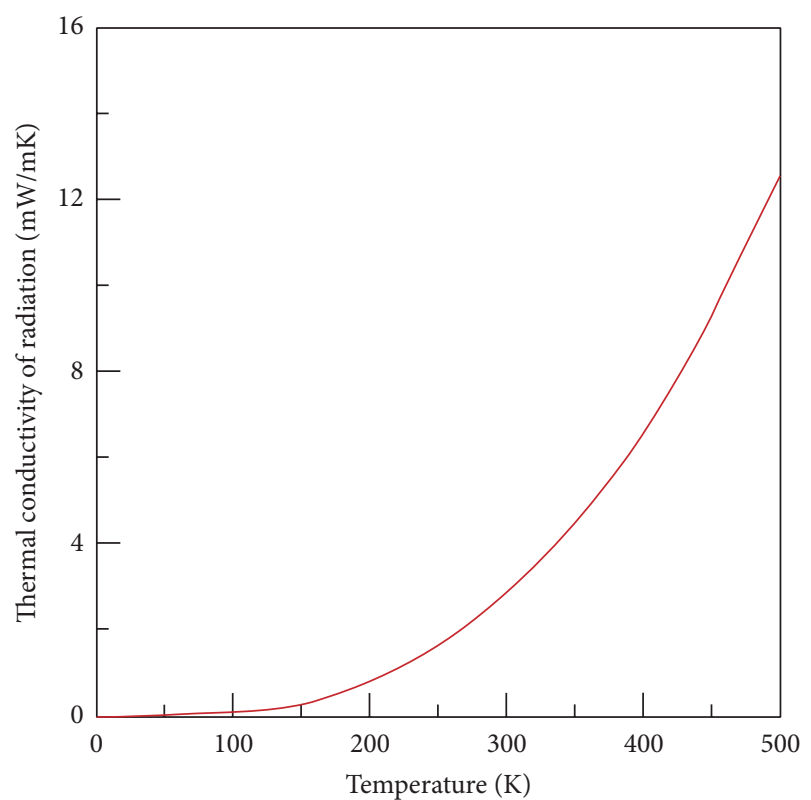

FIGURE 9: Relationship between the radiation thermal conductivity and temperature for gas-phase silica.

3.3. Radiation Heat Transfer. The following heat equation expresses radiation heat transfer in VIP [26]:

$$
q_{\mathrm{R}}=-\frac{16 n^{2} k_{\mathrm{B}} T^{3}}{3 K} \frac{d T}{d x}=-\frac{16 n^{2} k_{\mathrm{B}} T^{3}}{3 e(T) \rho} \frac{d T}{d x},
$$

where $K$ denotes the extinction coefficient of materials $\left(\mathrm{m}^{-1}\right)$, $K=e(T) \cdot \rho, e(T)$ denotes the specific extinction coefficient $\left(\mathrm{m}^{2} / \mathrm{kg}\right), \rho$ denotes the material density $\left(\mathrm{kg} / \mathrm{m}^{3}\right)$, and $n$ denotes the refractive index.

From the relationship between the heat flux and temperature gradient in (6), the thermal conductivity due to radiation heat transfer can be obtained [26]:

$$
\lambda_{\mathrm{R}}=\frac{16 n^{2} k_{\mathrm{B}} T^{3}}{3 K}=\frac{16 n^{2} k_{\mathrm{B}} T^{3}}{3 e(T) \rho} .
$$

Using gas-phase silica, Brodt [24] summarized the relationship between the radiation thermal conductivity and temperature, as shown in Figure 9. It can be seen from Figure 9 that when the temperature is below $150 \mathrm{~K}$, the radiation thermal conductivity is extremely small and can be neglected.

The addition of opacifiers to the core material can weaken the radiation heat transfer. Fricke noted that, at room temperature, the total thermal conductivity of pure silicon is $0.002-0.003 \mathrm{~W} /(\mathrm{m} \cdot \mathrm{K})$ higher than that of silicon with added opacifiers [7].

3.4. Convection. Once gases flow, heat will be transferred through convection. Convection is the transfer of heat from one place to another by the movement of gases or liquids. The most common convection medium in buildings is moist air. The penetration of moist air to enclosure structures is often 
TABLE 2: Comparison of the thermal performance of VIP and other thermal insulation materials [4].

\begin{tabular}{|c|c|c|c|c|c|c|c|}
\hline Materials & $\begin{array}{l}\text { Thermal conductivity } \\
\mathrm{mW} /(\mathrm{m} \cdot \mathrm{K})\end{array}$ & Cuttable? & $\begin{array}{l}\text { Fire resistance, } \\
\text { waterproofing, and } \\
\text { corrosion } \\
\text { resistance }\end{array}$ & $\begin{array}{c}\text { Prevention of } \\
\text { physical injuries }\end{array}$ & $\begin{array}{l}\text { Performance } \\
\text { after penetration }\end{array}$ & $\begin{array}{l}\text { Cost of unit } \\
\text { heat } \\
\text { resistance }\end{array}$ & $\begin{array}{l}\text { Environmental } \\
\text { impact }\end{array}$ \\
\hline VIP & $4-8$ & No & Low & Low & Weakened & High & Medium \\
\hline \multicolumn{8}{|c|}{ Conventional thermal insulation materials } \\
\hline Stone wool & $34-50$ & Yes & High & High & No change & Low & Low \\
\hline Glass fiber & $30-40$ & Yes & High & High & No change & Low & Medium \\
\hline Foam concrete & $70-80$ & Yes & High & High & No change & Low & Medium \\
\hline EPS & $30-40$ & Yes & Low & Medium & No change & Low & High \\
\hline XPS & $30-32$ & Yes & Medium & Medium & No change & High & High \\
\hline Polyurethane & $20-30$ & Yes & Medium & High & No change & High & High \\
\hline \multicolumn{8}{|c|}{ State-of-the-art insulation materials } \\
\hline Gas panel & $10-40$ & No & Low & Low & Weakened & High & Medium \\
\hline Aerogel & $13-14$ & Yes & Medium & Low & No change & High & Medium \\
\hline
\end{tabular}

Inorganic insulation materials
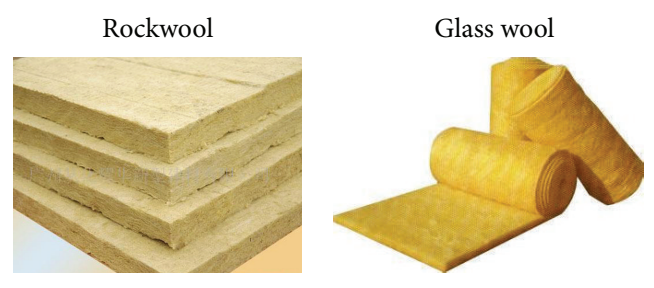

Expanded perlite

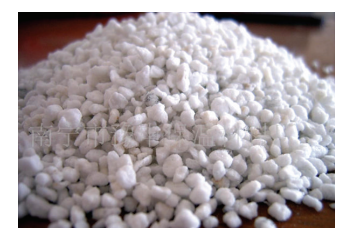

Calcium silicate

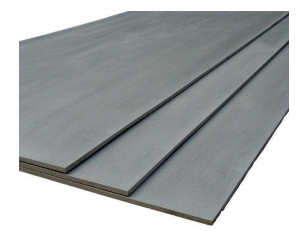

Aerated concrete

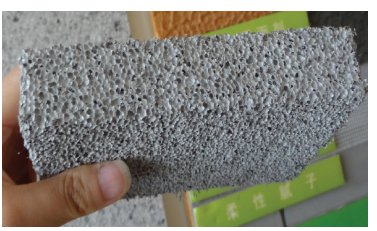

These materials are non-combustible and exhibit excellent fire resistance. However, their thermal conductivities are relatively high

Organic thermal insulation materials
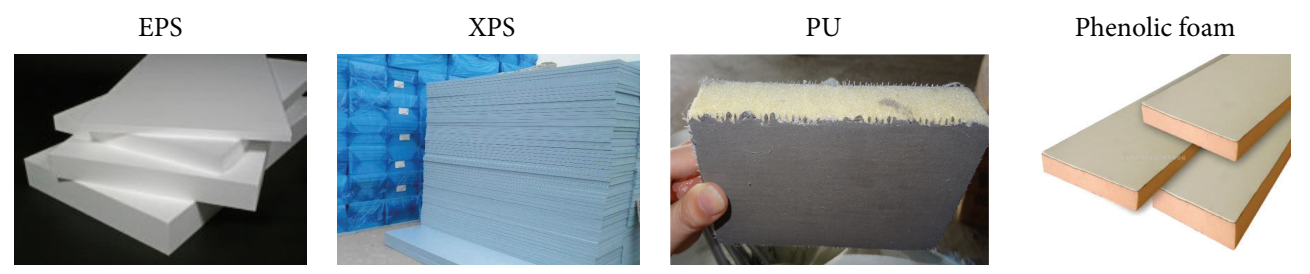

The thermal conductivities of these materials are low. However, the materials are flammable and exhibit poor

fire resistance

Figure 10: Classification of commonly used thermal insulation materials for buildings and their advantages and disadvantages.

accompanied by heat transfer. In addition, the heat transfer between the materials themselves and the surrounding air is normally conducted through convection.

Therefore, weakening of the gas thermal conductivity is the most effective way to reduce the total thermal conductivity. In VIP structure, by using membrane materials, the gas can be excluded from the main inner vacuum. This approach eliminates gas thermal conductivity.

\section{Application Potential of VIP in Chinese Buildings}

Currently, two types of thermal insulation materials are used to building walls, that is, inorganic and organic thermal insulation materials. Inorganic insulation materials include Rockwool, glass fiber, calcium silicate, and foam concrete, and organic insulation materials include expanded polystyrene (EPS), extruded polystyrene (XPS), and polyurethane (PU) foams. The thermal conductivity of inorganic thermal insulation materials is normally larger than that of organic thermal insulation materials, resulting in poorer insulation performance. However, the fire resistance performance of organic insulation materials is poor. Figure 10 and Table 2 list the thermal insulation materials on the market and their advantages and disadvantages. Therefore, compared with these materials, what is distinctive about VIP? The thermal performance, durability, physical properties, economy, and environmental impact of the materials are compared in Table 2. 
It can be seen from Table 2 that the thermal conductivity of VIP is much lower than that of other conventional insulation materials. According to the Chinese "design standard for energy efficiency of residential buildings in hot summer and cold winter zone," when the shape factor is $>0.4$, the total $U$-value of external walls is smaller than $0.8 \mathrm{~W} /$ $\left(\mathrm{m}^{2} \cdot \mathrm{K}\right)$ [27].

A Shanxi Datong residential building with a construction area of $100 \mathrm{~m}^{2}$ and a length and width of $10 \mathrm{~m}$ will be used as an example. In EnergyPlus, if the indoor electrical equipment and layout, people density, and timetable are same, a change in the wall insulation material from XPS to VIP of the same thickness will reduce the annual electricity consumption by $20.3 \%$, or the indoor net residential area can be increased by $2 \%$ while maintaining the same annual electricity consumption. Therefore, there is a huge potential for VIP application in high-performance buildings.

\section{Problems in the Application of VIP in Chinese Buildings}

The introduction, development, and application of VIP products have only been performed for approximately twenty years, and VIP research in China just began a few years ago. There are many theoretical investigations into the production performance, heat transfer, and aging of VIP. However, investigating VIP application in buildings is rare, and there are extremely few examples of the application of VIP to buildings worldwide. Although there is a huge potential for VIP application in Chinese buildings, many problems exist.

5.1. Failure by Puncturing. The VIP production process is complex, involving vacuum pumping and heat sealing. Therefore, once the products are molded, they cannot be cut. However, in the application process to real walls, it is difficult to manufacture a single-sized VIP for special positions, such as corners and surroundings of windows. Therefore, in the design stage, different VIP sizes are required. Compared with other cuttable materials, this aspect represents a major restriction on VIP applications. Additionally, during transportation, storage at a construction site, construction, and even delivery to use, the outer membranes of VIP can be easily punctured and thus cause the loss of the vacuum, which greatly increases the thermal conductivity of VIP. Binz et al. [11] reported in 2005 that, given that the surface of VIP can be easily punctured and lose its vacuum, the thermal conductivity of a punctured VIP is 5 times that of an intact VIP. However, most VIP constructions currently are mounted on the building sites. The storage at construction sites is random and chaotic; therefore, there exist many unpredictable factors that can easily puncture the VIP, leading to the loss of its function. Figure 11 shows the storage of VIP at a construction site, and Figure 12 shows the comparison of VIP before and after puncture.

5.2. Heat Bridge. When VIP performance is discussed, normally, only the thermal conductivity at the center of the panels has been taken into account. However, in real applications, it is more appropriate to consider the effective

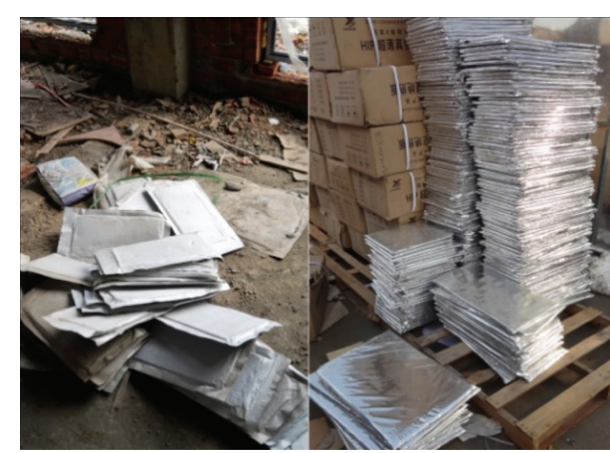

FIGURE 11: VIP at construction site.

thermal conductivity by taking into account the heat bridge effects surrounding the VIP. In real applications, a heat bridge can be observed with three layers, that is, (1) VIP layer, (2) building component layer, and (3) building façade layer [28]. The heat bridge of a VIP layer is induced by the huge difference in thermal conductivity between the vacuumed core material and outer membrane, as shown in Figure 13.

The linear thermal conductivity of the VIP boundary is related to the thickness, circumference, and surface area of the panels. The effective thermal conductivity of a VIP can be calculated with the following equation [29]:

$$
\lambda_{\text {eff }}=\lambda_{\text {cop }}+\Psi_{\text {VIP edge }} \cdot d_{p} \cdot \frac{l_{p}}{S_{p}},
$$

where $\lambda_{\text {cop }}$ denotes the thermal conductivity of the VIP panel center $(\mathrm{W} /(\mathrm{m} \cdot \mathrm{K})), \Psi_{\mathrm{VIP}}$ edge denotes the linear thermal conductivity $(\mathrm{W} /(\mathrm{m} \cdot \mathrm{K})), \lambda_{\text {eff }}$ denotes the effective thermal conductivity, $d_{p}$ denotes the thickness of the VIP (m), $l_{p}$ denotes the circumference of boundaries, and $S_{p}$ denotes the surface area.

It can be seen from Figure 14 that because the VIP sizes cannot be large, many VIPs must be combined for the façade of the entire building, resulting in a large number of joints. The heat bridge effects at the joints on the entire walls cannot be ignored.

5.3. VIP Cannot Be Anchored and Perforated. Currently, the thermal insulation of the outer walls by VIP requires bonding or a combination of bonding and anchorage. For high-rise buildings, due to the large area, the combination of bonding and anchorage is commonly used, as shown in Figures 15 and 16. Insulation nails are used to secure the joining areas of four adjacent VIPs.

Because a VIP cannot be perforated, the anchorage position cannot be as flexible as the conventional insulation materials. Because the anchorage is implemented at the boundaries, it will result in larger gaps between adjacent VIPs and thus create more heat losses.

In addition, there are many openings on the wall, for example, air louvers, entrance holes for electrical lines and water pipelines, and drainage holes. These positions will cause much difficulty in the application of VIP. Therefore, some parts of the building envelopes still need to be done with other cuttable insulation materials. 


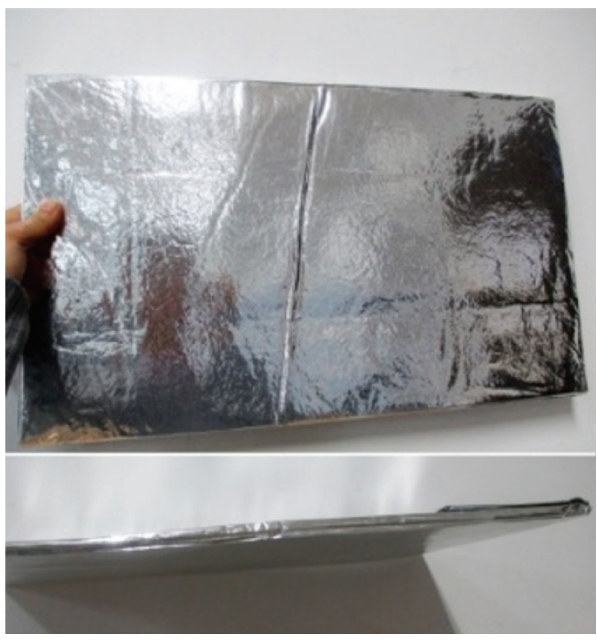

(a) Before loss of vacuum

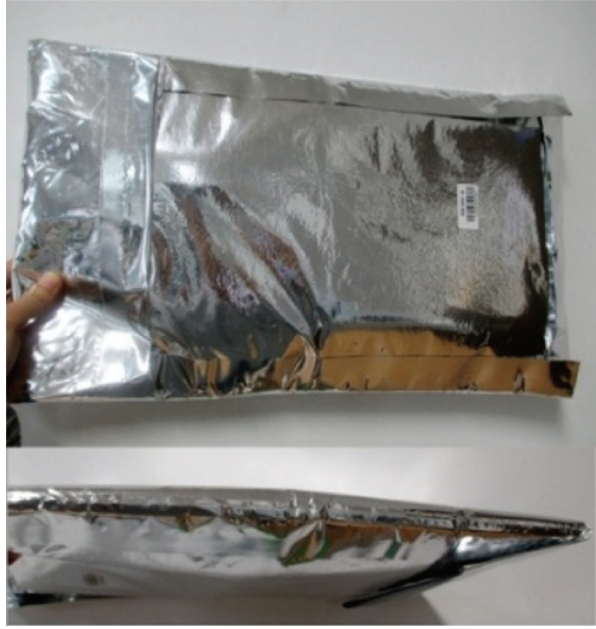

(b) After loss of vacuum

FIGURE 12: Comparison of VIP before and after puncture and loss of vacuum in China.

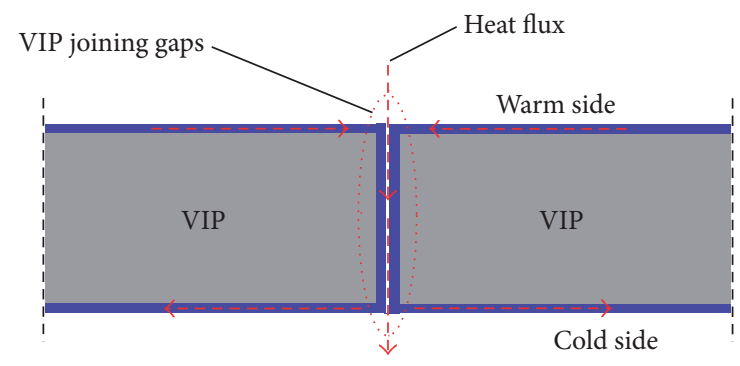

FIGURE 13: Heat bridge effect surrounding the VIP.

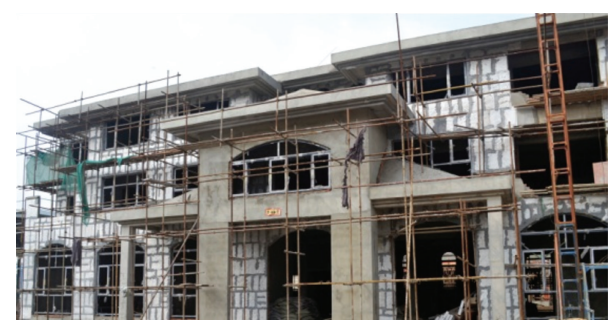

Figure 14: VIP at the façade of building.

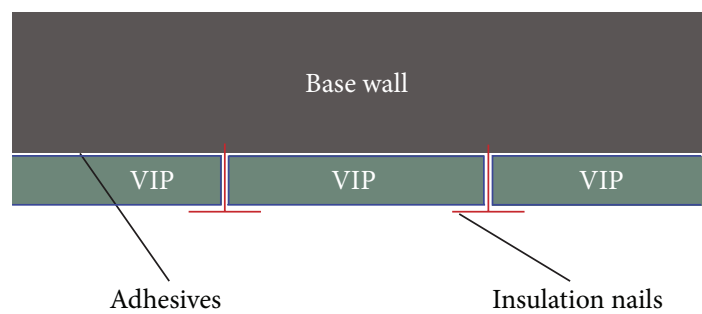

FIGURE 15: Anchorage of VIP.

When VIPs are used as insulation materials inside walls, the problems associated with nonanchorage and nonperforation become more pronounced. Once construction is

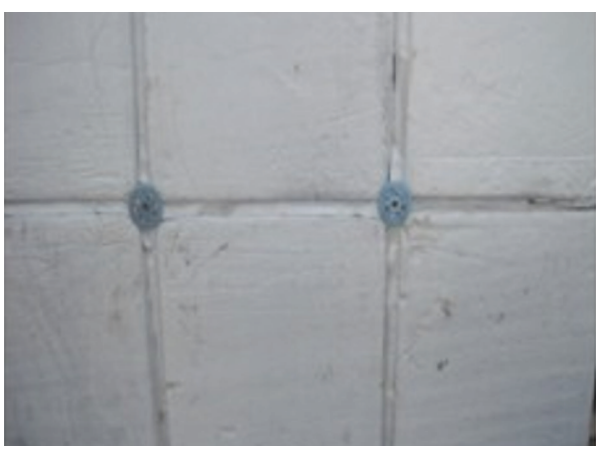

FIGURE 16: Actual images of VIP anchored by insulation nails.

complete, no nails can be applied to the surface of the entire wall for a suspended wall cupboard, household appliances, and hooks; these basic setups cannot be avoided in Chinese homes. Particularly, after a long period of time or a change of ownership, these problems will become more obvious for the second interior decoration.

Boafo et al. proposed an improved solution which might be possible to address these problems. Figure 17 described a sectional view of an insulated wall system showing the material layers [30].

VA-Q-TEC [31] proposed a solution, as shown in Figure 18. During VIP production, circular, semicircular, or unusually shaped holes are reserved. In those special locations on the wall, these VIP products with optimized shapes for special needs can be used to complement the aforementioned regular VIP.

However, due to the exceptionally low thermal conductivity, only extremely thin VIP will be able to meet requirements in real applications. Therefore, their thermal insulation capability is lost in those anchorage holes, resulting in severe heat bridges. As such, the usage of these VIPs with holes or opening requires compromise. These VIPs can only 


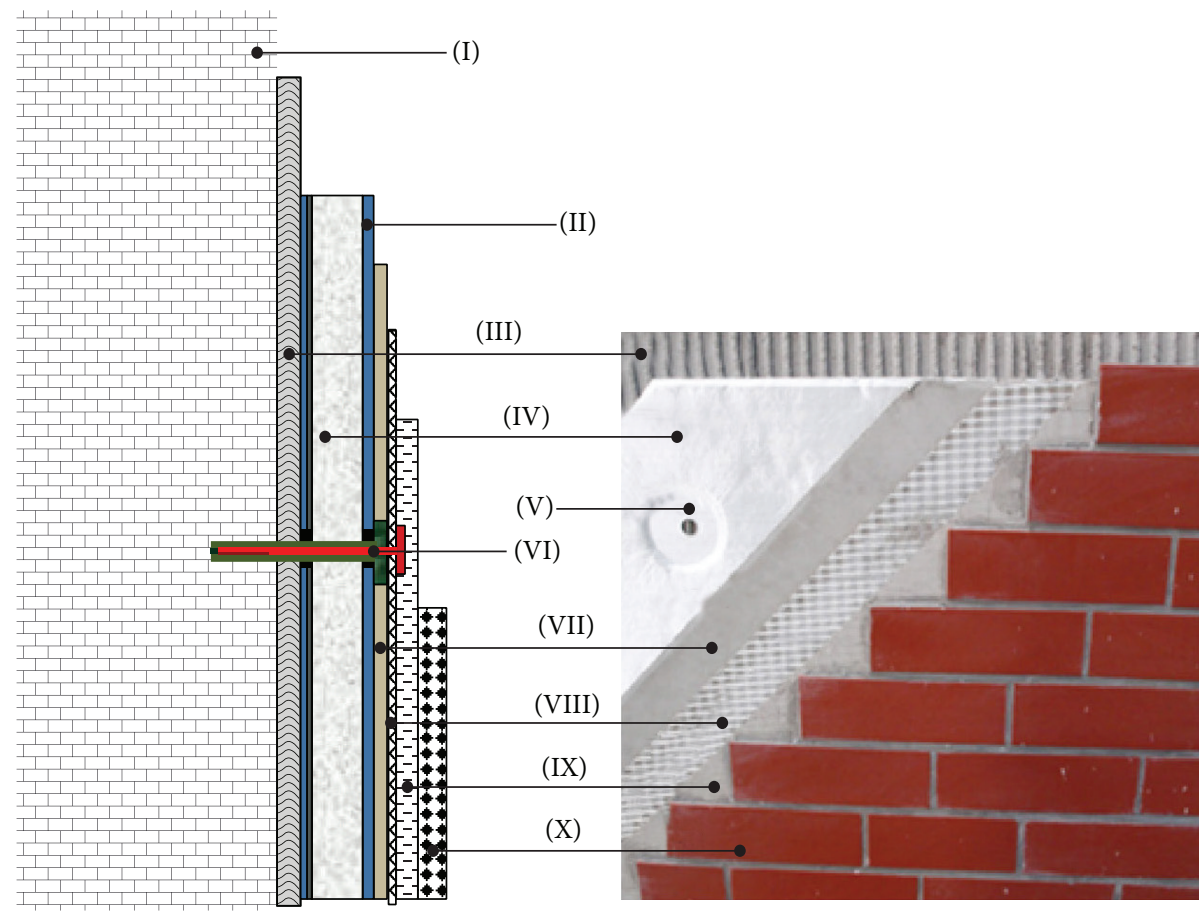
(I) Existing wall
(II) Interface adhesive powder
(III) Bonding mortar (with gutters)
(IV) Vacuum insulation panel
(V) Hole of support

(a)
(VI) Plastic spacer

(VII) Plastic mortar

(VIII) Fiberglass mesh

(IX) Surface mortar plaster

(X) Surface decorations

Figure 17: Assembly of vacuum insulation system: (a) cross section and (b) pictorial view of insulated wall [30].
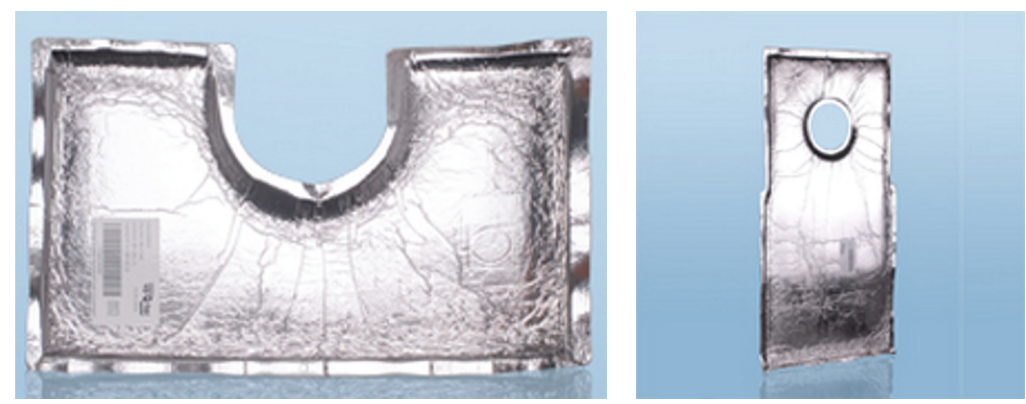

FIGURE 18: VIP with holes or opening.

be used in the places that require them, such as air louvers and line and wire holes.

\section{Conclusions}

The selection of VIP core materials, membranes, and their construction is based on certain thermal insulation mechanism. Inner core materials with porosity, excellent frame geometrics, and lightweight, such as glass fiber and silica, can effectively reduce solid heat transfer. High porosity ensures that the inner part can be vacuumed, whereas the membrane will ensure the maintenance of the high vacuum inside, which essentially prevents gas convection from occurring inside the material. The metal foils and multilayered metallized polymer membranes can maximally reduce the gas penetration to the inside and the loss of vacuum; therefore, the reduction of gas thermal conductivity is further enhanced. The getters inside the VIP can collect and remove gases either leaked through 
the membrane or off gassed from the membrane materials over time. The low thermal conductivity of VIP is attributed to the reduction of thermal conductivity and radiation.

In real buildings, due to the low thermal conductivity, extremely thin VIP will be able to meet the standard requirements. This capacity will drastically reduce the wall thickness and increase the indoor usage area. If the same thickness of VIP and conventional insulation materials are used, the usage of VIP will drastically reduce energy consumption from air conditioning in buildings.

However, at present, several problems exist in the application of VIP in Chinese buildings.

(1) Failure: the membrane of a VIP can be easily damaged by puncturing, ripping, or squeezing, resulting in vacuum leakage and a drastic decrease in the thermal insulation performance.

(2) Heat bridge: because the VIP membrane contains a foil layer, such as aluminum foil, heat is easily transferred at the boundaries of the VIP panels, which creates natural heat bridges.

(3) Noncuttability: the size of the VIP panels cannot be changed once manufactured, and the panels cannot be cut on-site according to real applications. As a result, arranging VIP on walls becomes a complex and difficult task.

(4) Nonanchorage and nonperforation: in the construction process, VIP cannot be perforated. As a result, the means to apply VIP to walls are extremely limited.

Overall, VIPs are thermal insulation materials with distinct advantages and disadvantages. If not appropriately used, their advantages cannot be fully utilized, and their disadvantages will dominate. Problems exist for VIP. If the problems are addressed alone, other problems could result. Therefore, the VIP thermal insulation system should be considered as an integral part. The material, structure, system, and their relationship must be systematically considered. Based on the real conditions and different building types, the problem must be addressed systemically to provide solutions.

\section{Competing Interests}

The authors declare that they have no competing interests.

\section{Acknowledgments}

The research presented in this paper was supported by the National Natural Science Foundation of China (51278107), China Scholarship Council (201406095032), Top-Notch Academic Programs Project of Jiangsu Higher Education Institutions, the Key Program of the Natural Science Foundation of Jiangsu Province (BK2010061), the R\&D Program of the Ministry of Housing and Urban-Rural Development of China (2011-K1-2), the Open Project Program of the Key Laboratory of Urban and Architectural Heritage Conservation (Southeast University), and the Ministry of Education (KLUAHC1212).

\section{References}

[1] https://en.wikipedia.org/wiki/Beijing_Television_Cultural_ Center_fire.

[2] March 2016, https://en.wikipedia.org/wiki/2010_Shanghai_fire.

[3] American Society for Testing and Mateirals, "Standard specification for vacuum insulation panels," ASTM C1484-10, American Society for Testing and Mateirals, West Conshohocken, Pa, USA, 2010.

[4] M. Erb and W. Symons, "Vacuum insulation panel properties and building applications," ECBCS Annex 39 project summary report. 2010.

[5] J. Fricke, "From Dewars to VIPs-one century of progress in vacuum insulation technology," in Proceedings of the 7th International Vacuum Insulation Symposium, M. Zimmerman, Ed., pp. 5-14, EMPA, September 2005.

[6] 2016, http://vip-bau.de/e_pages/monitoring/monitoring.htm.

[7] J. Fricke, "Physical aspects of heat transfer and the development of thermal insulations and Materna R, Wancor ZZ. VIP's for advanced retrofit solutions for buildings," in Proceedings of the International Conference and Workshop, High Performance Thermal Insulation Systems Vacuum Insulated Products (VIP), pp. 13-22, Duebendorf, Switzerland, January 2001.

[8] S. E. Kalnæs and B. P. Jelle, "Vacuum insulation panel products: a state-of-the-art review and future research pathways," Applied Energy, vol. 116, pp. 355-375, 2014.

[9] I. Mandilaras, I. Atsonios, G. Zannis, and M. Founti, “Thermal performance of a building envelope incorporating ETICS with vacuum insulation panels and EPS," Energy and Buildings, vol. 85, pp. 654-665, 2014.

[10] P. Johansson, C.-E. Hagentoft, and A. S. Kalagasidis, "Retrofitting of a listed brick and wood building using vacuum insulation panels on the exterior of the facade: measurements and simulations," Energy and Buildings, vol. 73, pp. 92-104, 2014.

[11] A. Binz, A. Moosmann, G. Steinke et al., "Vacuum insulation in the building sector. Systems and applications (subtask B)," Final Report for the IEA/ECBCS Annex 39, 2005.

[12] Ministry of Housing and Urban-Rural Development of the People's Republic of China (MOHURD), Vacuum Insulation Panels for Buildings (JG/T 438-2014), The IEA/ECBCS Annex, China Architecture \& Building Press, Beijing, China, 2005, [In Chinese].

[13] Th. Thorsell, Advances in thermal insulation-vacuum insulation panels and thermal efficiency to reduce energy usage in buildings [Ph.D. thesis], 2012.

[14] J.-S. Kwon, C. H. Jang, H. Jung, and T.-H. Song, "Effective thermal conductivity of various filling materials for vacuum insulation panels," International Journal of Heat and Mass Transfer, vol. 52, no. 23-24, pp. 5525-5532, 2009.

[15] R. De Vos, D. Rosbotham, and J. Deschaght, "Open-celled polyurethane foam based vacuum panel technology: a fully polyurethane based composite technology for vacuum insulated appliances," Journal of Cellular Plastics, vol. 32, no. 5, pp. 470484, 1996.

[16] B. P. Jelle, A. Gustavsen, and R. Baetens, "The path to the high performance thermal building insulation materials and solutions of tomorrow," Journal of Building Physics, vol. 34, no. 2, pp. 99-123, 2010.

[17] March 2016, http://www.thomasnet.com/articles/materialshandling/fiberglass-safety-health-concerns. 
[18] H. Li and L. Liu, "The impact of production and filling technology of expanded perlite on its insulating properties," Cryogenic Technology, no. 3, pp. 33-34, 2002 (Chinese).

[19] P. Mukhopadhyaya, K. Kumaran, N. Normandin, D. van Reenen, and J. Lackey, "High-performance vacuum insulation panel: development of alternative core materials," Journal of Cold Regions Engineering, vol. 22, no. 4, pp. 103-123, 2008.

[20] B. P. Jell and S. E. Kalnæs, "Nanotech-based vacuum insulation panels for building applications," in Nano and Biotech Based Materials for Energy Building Efficiency, P. Torgal, C. Buratti, S. Kalaiselvam, C.-G. Granqvist, and V. Ivanov, Eds., pp. 167-214, Springer, Berlin, Germany, 2016.

[21] M. Alam, H. Singh, and M. C. Limbachiya, "Vacuum insulation panels (vips) for building construction industry-a review of the contemporary developments and future directions," Applied Energy, vol. 88, no. 11, pp. 3592-3602, 2011.

[22] S. Brunner, P. Gasser, H. Simmler, and K. Ghazi Wakili, "Investigation of multilayered aluminium-coated polymer laminates by focused ion beam (FIB) etching," Surface and Coatings Technology, vol. 200, no. 20-21, pp. 5908-5914, 2006.

[23] J. Fricke, "Materials research for the optimization of thermal insulations," High Temp-High Press, vol. 25, pp. 379-390, 1993.

[24] K. Brodt, Thermal insulations: Cfc-alternatives and vacuum insulation [Ph.D. thesis], Delft University Press, Delft, Netherlands, 1995.

[25] M. G. Kaganer, Thermal Insulation in Cryogenic Engineering, Translated by A. Moscona, Israel Programme for Scientific Translation, 1969.

[26] F. Kreith and W. Z. Black, Basic Heat Transfer, Harper \& Row, New York, NY, USA, 1980.

[27] Ministry of Housing and Urban-Rural Development of the People's Republic of China (MOHURD), Design Standard for Energy Efficiency of Residential Buildings in Hot Summer and Cold Winter Zone in China (JGJ134-2010), China Architecture \& Building Press, Beijing, China, 2010.

[28] D. Quenard and H. Sallee, "From VIP's to building facades: three levels of thermal bridges," in Proceedings of the 7th International Vacuum Insulation Symposium, M. Zimmermann, Ed., pp. 113-120, Duebendorf, Switzerland, 2005.

[29] K. Ghazi Wakili, R. Bundi, and B. Binder, "Effective thermal conductivity of vacuum insulation panels," Building Research and Information, vol. 32, no. 4, pp. 293-299, 2004.

[30] F. E. Boafo, Z. Chen, C. D. Li, B. Li, and T. Xu, "Structure of vacuum insulation panel in building system," Energy and Buildings, vol. 85, pp. 644-653, 2014.

[31] http://www.va-q-tec.com/. 

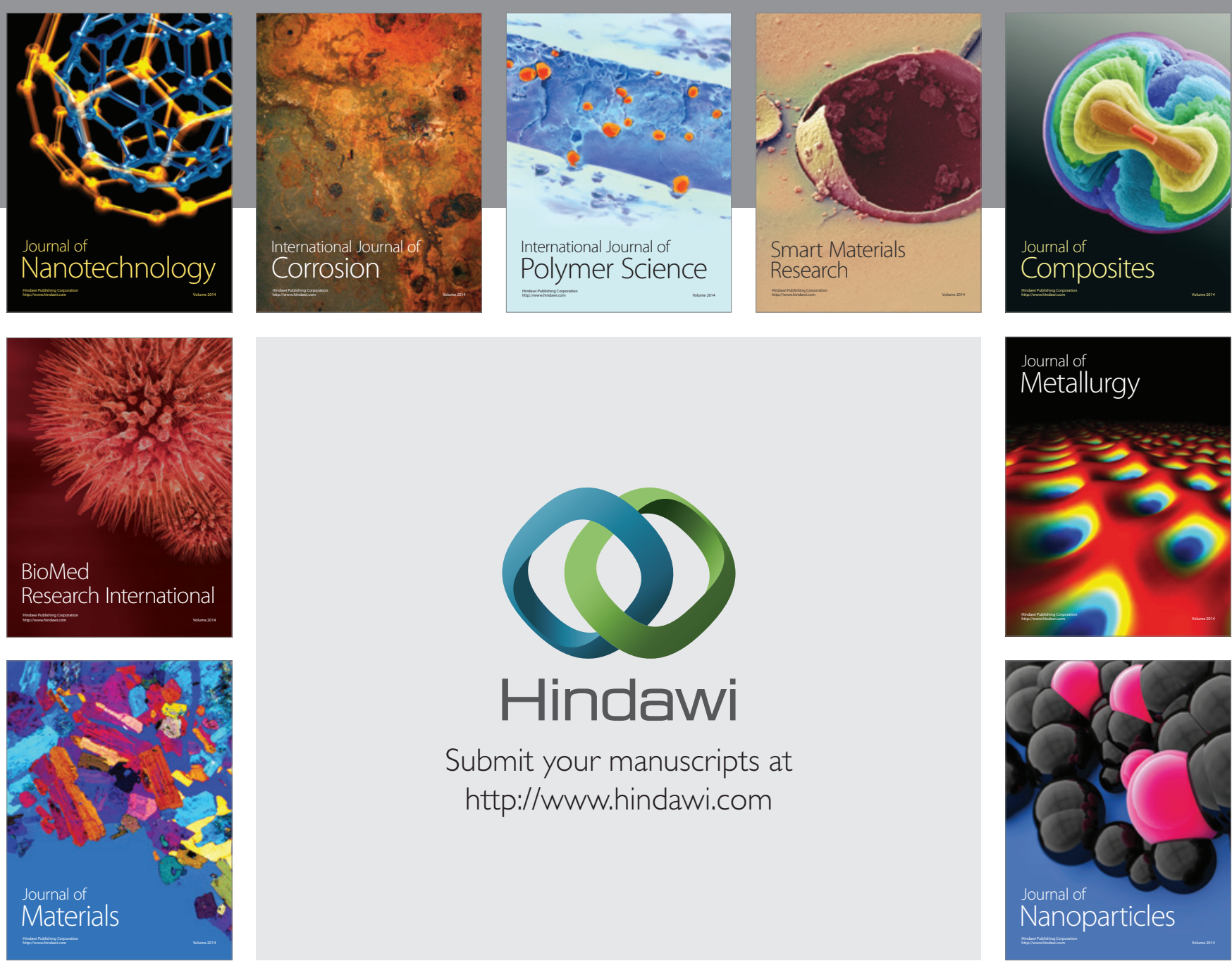

\section{Hindawi}

Submit your manuscripts at

http://www.hindawi.com

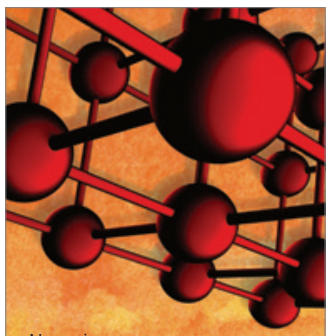

Materials Science and Engineering
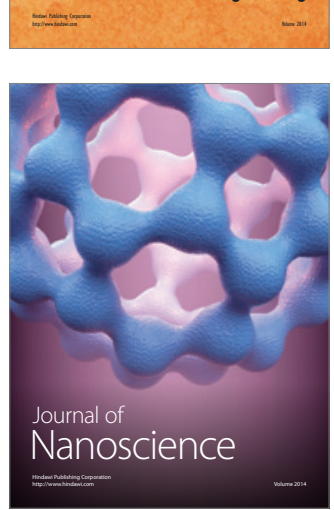
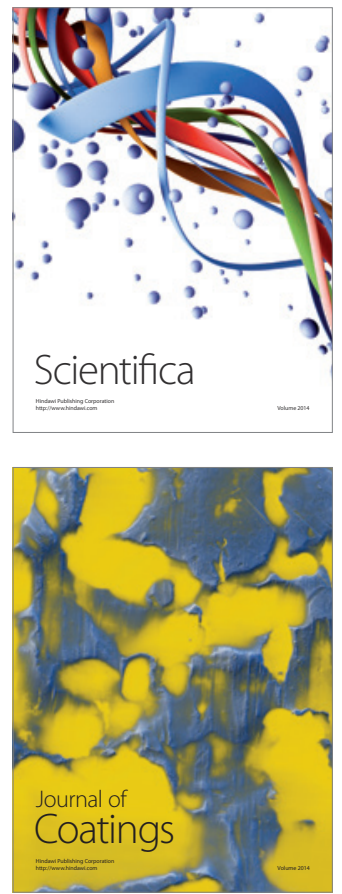
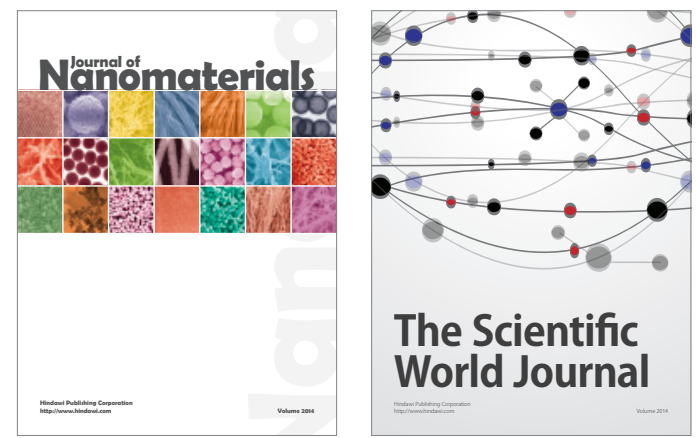

The Scientific World Journal
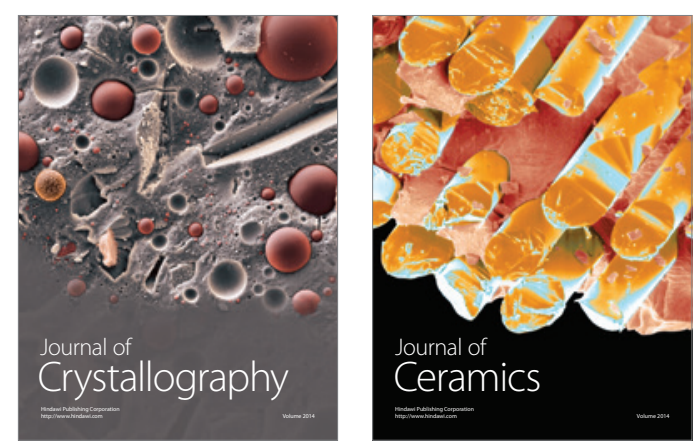
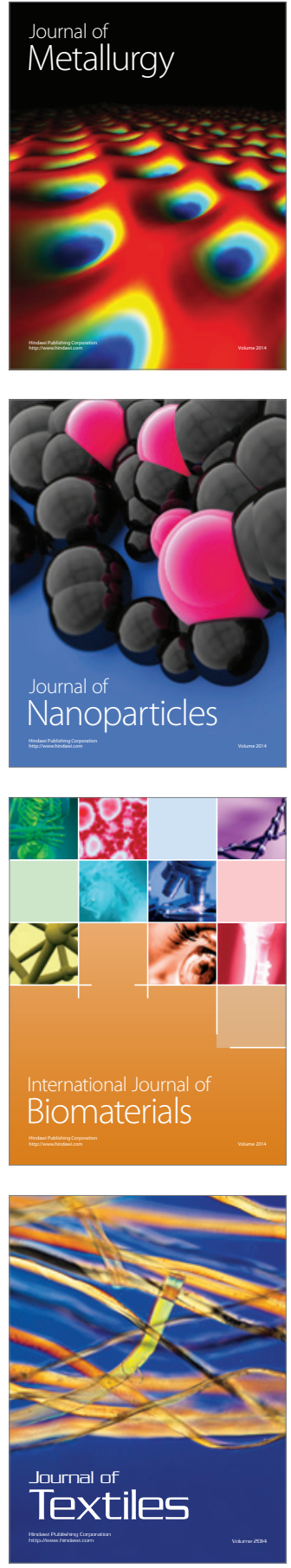